\title{
Optimisation of ultrasound-assisted extraction of oil from papaya seed by response surface methodology: Oil recovery, radical scavenging antioxidant activity, and oxidation stability
}

\begin{abstract}
The present study aimed to investigate the effects of ultrasound-assisted extraction (UAE) condition on the yield, antioxidant activity and stability of the oil from papaya seed. The studied ultrasound variables were time, temperature, ultrasound power and solvent to sample ratio. The main goal was to optimise UAE condition providing the highest recovery of papaya seed oil with the most desirable antioxidant activity and stability. The interaction of ultrasound variables had the most and least significant effects on the antioxidant activity and stability, respectively. Ultrasound-assisted extraction provided a relatively high oil recovery $(\sim 73 \%)$ from papaya seed. The strongest antioxidant activity was achieved by the extraction at the elevated temperature using low solvent to sample ratio. The optimum ultrasound extraction was set at the elevated temperature $\left(62.5^{\circ} \mathrm{C}\right)$ for $38.5 \mathrm{~min}$ at high ultrasound power $(700 \mathrm{~W})$ using medium solvent to sample ratio $(\sim 7: 1 \mathrm{v} / \mathrm{w})$. The optimum point was practically validated.
\end{abstract}

Keyword: Ultrasound-assisted extraction; Radical scavenging antioxidant activity; Papaya seed oil; Oil stability; Extraction yield 